\title{
A Sero-epidemiological Survey of Brucellosis, Q-Fever and Leptospirosis in Livestock and Humans and Associated Risk Factors in Kajiado County- Kenya
}

\author{
Nakeel MJ*, Arimi SM, Kitala PK, Nduhiu G, Njenga JM and Wabacha JK \\ Department of Public Health, Pharmacology and Toxicology, Kangemi, Kenya
}

*Corresponding author: Nakeel MJ, University of Nairobi, College of Agriculture and Veterinary Sciences, Department of Public Health, Pharmacology and Toxicology, Kangemi, Kenya, E-mail: info@nakheel.com

Received date: June 7, 2016; Accepted date: June 16, 2016; Publication date: June 22, 2016

Copyright: (C) 2016 Nakeel MJ, et al. This is an open-access article distributed under the terms of the Creative Commons Attribution License; which permits unrestricted use; distribution; and reproduction in any medium; provided the original author and source are credited.

\begin{abstract}
Animal and human health is inextricably linked. People depend on animals for nutrition, socio-economic development and companionship. A cross-sectional serological study was carried out to determine the seroprevalence of three related zoonotic diseases namely Brucellosis, Q-fever and Leptospirosis in cattle, sheep, goats and humans in three sub counties of Kajiado County. In addition the risk factors associated with sero-positivity in animals and humans were assessed. A total of, 250 (cattle), 167 (sheep), (167) goats and 317 (humans) samples were collected. Serum samples were screened for brucellosis using Rose Bengal Plate Test (RBPT) and thereafter a total of 400 samples from all the four species (all the positive on RBPT and other randomly picked samples) were subjected to cELISA (COMPELISA, VLA, UK) test. Results indicated a low prevalence of brucellosis in humans $1.3 \%(2 / 150)$ but a higher prevalence of $\mathrm{Q}$-fever $26 \%(24 / 90)$. The overall prevalence in livestock was $12.91 \%$ $(27 / 209)$ and $79.3 \%$ (249/314) $21.8 \%$ (54/248) (in cattle only) for brucellosis, Q-fever and Leptospirosis respectively. The prevalence estimates in cattle, sheep and goats were $21.92 \%(16 / 73), 8.6 \%(6 / 69)$ and $7.3 \%(5 / 67)$ for brucellosis respectively and $89.7 \%(140 / 156), 57.5 \%$ (46/80) and $83.1 \%(69 / 83)$ for Q-fever respectively and $21.8 \%$ $(54 / 248)$ for Leptospirosis in cattle only, indicating a high risk of transmission of the diseases to humans through contact and/or consumption of livestock products such as milk. The study and the data obtained therefore indicates that the two zoonotic diseases brucellosis and Q-fever maybe enzootic in the study area in human, cattle, sheep and goats, while leptospirosis is present in cattle and presents a serious public health problem among the inhabitants of the county and that there is need to create awareness among all concerned on the likely high prevalence of the two diseases to avoid misdiagnosis and suffering of patients. It is recommended that the veterinary personnel in Kajiado County make an effort to investigate all cases of abortions and retained placentas that are included in their disease surveillance reports.
\end{abstract}

Keywords: Interstitial nephritis; Uveitis; Tubulointerstitial nephritis; Uveitis syndrome; Autoimmunity

\section{Introduction}

Zoonosis or diseases transmitted from animals to man, have been recognized as important public health issues for centuries and much of the early history of veterinary science was focused on the control of diseases such as bovine tuberculosis. Ungulates, in particular, are known to carry at least 315 zoonotic pathogens [1] and many emerging and re-emerging infectious disease problems globally are zoonotic [2]. In spite of the clear need to understand these diseases in the animal populations where they may be maintained [3] the veterinary and medical professions need to work closely on infectious disease research in multidisciplinary teams to be successful in tackling many of these diseases. There is a clear and urgent need for this in subSaharan Africa (SSA) where the public health and veterinary infrastructures have virtually collapsed through neglect and enforced privatization. Brucellosis, Q-fever and Leptospirosis are important bacterial infections of livestock causing a range of clinical conditions that lead to infertility and loss of production; they are also found in wildlife. In addition, they are important zoonotic infections affecting people who work with livestock or consume contaminated animal products such as milk. The diseases produce non-specific symptoms including fever, which are often misdiagnosed leading to severe chronic disease [4].

The economic importance and public health significance of brucellosis cannot be over emphasized. The disease is widely distributed in Africa with the highest incidence in areas where extensive livestock husbandry is practised and animal populations are high [5]. It is transmitted directly or indirectly from infected animals to man with consequent debilitation and prolonged incapacitation. Six hundred and nine (609) cases based on Rose Bengal Plate Test (RBPT) of human brucellosis have been reported in Kajiado County [6]. However RBPT is a screening test that is prone to giving false positives. Similarly eight hundred and ninety seven (897) cases of animal Brucellosis have been reported from 2003- 2011 in the whole country based on Rose Bengal Plate test (RBPT), Enzyme Linked Immunosorbent Assay (ELISA) and Complement Fixation Test (CFT) at the Central Veterinary Laboratories in Kabete. Eleven (11) cases were confirmed in Kajiado East Sub County. However, cases of unspecified abortion and retained afterbirths were reported during that period in Kajiado County. Information regarding the prevalence of brucellosis in the county is scanty and disjointed. It is known that animals act as reservoirs for human infections and therefore it is necessary to carry out surveys to ascertain the prevalence of this disease, which causes serious economic losses. Real risk prevails [7] but there are no records of any surveys conducted in the County. 
Citation: Nakeel MJ, Arimi SM, Kitala PK, Nduhiu G, Njenga JM, et al. (2016) A Sero-epidemiological Survey of Brucellosis, Q-Fever and Leptospirosis in Livestock and Humans and Associated Risk Factors in Kajiado County-Kenya. J Trop Dis 4: 215. doi: 10.4172/2329-891X.1000215

Page 2 of 8

Q-fever is a highly contagious zoonotic disease with multiple hosts that can serve as reservoirs of infection, but aborting domestic ruminants are typically the main source of the bacterium in humans and other animals [8]. Early studies done in Nyanza, Coast and Central Kenya from 1955 to 1976 among domestic ruminants showed the prevalence of antibodies was reported as $7-57 \%$ in cattle and $33-34 \%$ in goats. A more recent study done in 2007 to 2008 in cattle, sheep and goats in Western Kenya the prevalence of antibodies against C.burnetii was reported as $28.3 \%$ in cattle, $32 \%$ in goats, and $18.2 \%$ in sheep [9].

Despite the high-profile nature of some Q-fever outbreaks, and the attention that C.burnetii has received as potential bioterrorism agent [10] information on the prevalence of infection in sub-Saharan Africa is scanty [11]. In Kenya, serological evidence of Q-fever in patients with acute febrile and respiratory illness was shown in the 1950s. Other studies between 1956 and 1976 showed the prevalence of antibodies to C.burnetii among Kenyans to range between $10 \%$ and 20. A more recent investigation found that four people (8\%) of a group of 50 travellers to Kenya contracted Q-fever and in another recent study investigators diagnosed acute Q-fever in 5\% of febrile patients admitted to two hospitals from September 2007 to August 2008, in neighbouring northern Tanzania [12]. The latest study done in Western Kenya in 2009 to 2010 reported a prevalence of $30.9 \%$ in human sera that were collected between 2007-2008 [9].

Leptospirosis is a zoonosis of ubiquitous distribution, caused by infection with pathogenic spirochetes belonging to the genus Leptospira. They infect a wide spectrum of hosts, including mammals, reptiles, birds and amphibians. They pose a significant public health problem of increasing concern as well as great impact on the reproductive efficiency of livestock [13]. Cattle are the maintenance host for Leptospira borgpetersenii serovar Hardjo (subtype hardjobovis) and Leptospira interrogans serovar Hardjo (subtype hardjoprajitno), which are serologically indistinguishable but genetically distinct [13]. A variety of clinical illnesses are seen when a cow becomes infected for the first time: abortion, mastitis, loss of milk and calves may be stillborn, weak or clinically normal but infected. Infertility associated with persistent infection is the most important economic consequence. Infection is usually transmitted directly by contact with infected urine, run-off water or abortion fluids from infected animals. The situation regarding Leptospirosis in Africa is mostly unknown and rarely documented outside South Africa [14] although it is associated with high rainfall regions in cattle in South Africa. Symptoms of Leptospirosis in man include high fever, severe headache, chills, muscle aches, and vomiting, and may include jaundice, red eyes, abdominal pain, diarrhoea, and/or a rash. The symptoms in humans appear after a 14 day incubation period following contact with infected urine from animals.

This study in Kajiado County provided a valuable opportunity for generating domestic animal prevalence data that could be linked to human health outcomes. To assess the current status of Q-fever among humans and infection prevalence in domestic ruminants, surveys were conducted for C.burnetii in cattle, goats, sheep, and tested specimens from human patients presenting to a clinic in the same area. The size and extent of these three zoonotic diseases in the Kajiado ecosystem remains largely unknown. It was therefore necessary to conduct investigations aimed at establishing their status in order to lessen the potential negative impacts they exert in the ecosystem. In this study the estimation of the prevalence of brucellosis and Q-fever and Leptospirosis was made in cattle, sheep, goats and humans in three Sub- Counties that make a transect from east to west of Kajiado County. The County is inhabited mainly by the Maasai community which still practices nomadic pastoralism to a large extent. Like other pastoralists, livestock play an important central role in their daily and ceremonial life. They depend on livestock for meat, milk and blood in addition to providing the principal currency for social and commercial transactions.

The overall objective of this study was to estimate the seroprevalence of Brucellosis and Q-fever and Leptospirosis to establish disease implications on the pastoral community in Kajiado County with the specific objectives being, to estimate the sero-prevalence of Brucellosis, Q-fever and Leptospirosis in livestock and humans and to determine the risk factors associated with the occurrence in livestock and humans. This paper presents a serological analysis of exposure to Brucella species, Coxiella burnetti Leptospira species, in cattle, sheep, goats and humans in three areas of Kajiado County in Kenya from July to September 2012. Historical data of patient records in the three health facilities were compiled for the six years. Then a cross sectional survey was conducted to sample livestock in the three divisions to determine sero-reactors to the three diseases brucellosis and Q-fever and Leptospirosis (where they were available) and the risk factors associated with these zoonosis. Patients seeking medical attention in the three health facilities and presenting with flu-like symptoms in the course of the study were also sampled.

\section{Materials and Methods}

\section{Sampling humans}

Visits were made to the three health facilities and any patients presenting with flu-like symptoms were recruited upon consent into the study. The hospital clinicians were in-charge of examining the patients, identifying the cases with flu-like symptoms, seeking their consent to participate in the study and taking of blood samples for testing for the presence of the two zoonoses. After normal history taking and responding to the questionnaire the qualifying patients were sent to the laboratory for blood sampling by the hospital staff. About $5-10 \mathrm{ml}$ of blood was collected aseptically in plain vacutainers. A total of two hundred and fifty 250 samples were collected. Map of the whole Kajiado County and the study sites is shown in (Figure 1).

\section{Livestock}

With the assistance of the veterinary staff, herds from areas identified based on suspected cases of abortion were identified and sampled. The herds were selected based on their accessibilities and other logistics. Animals (Cattle, Sheep, and Goats) within selected herds that were six months old and above were sampled using a systematic random method until the required sample size was achieved. $10 \mathrm{mls}$ of blood was collected Plain vacationers by bleeding the animals from the jugular vein following restraint and each sample labelled immediately after collection. A total of 237 cattle, 167 sheep and 167 goat samples were collected. 
Citation: Nakeel MJ, Arimi SM, Kitala PK, Nduhiu G, Njenga JM, et al. (2016) A Sero-epidemiological Survey of Brucellosis, Q-Fever and Leptospirosis in Livestock and Humans and Associated Risk Factors in Kajiado County-Kenya. J Trop Dis 4: 215. doi: 10.4172/2329-891X.1000215

Page 3 of 8
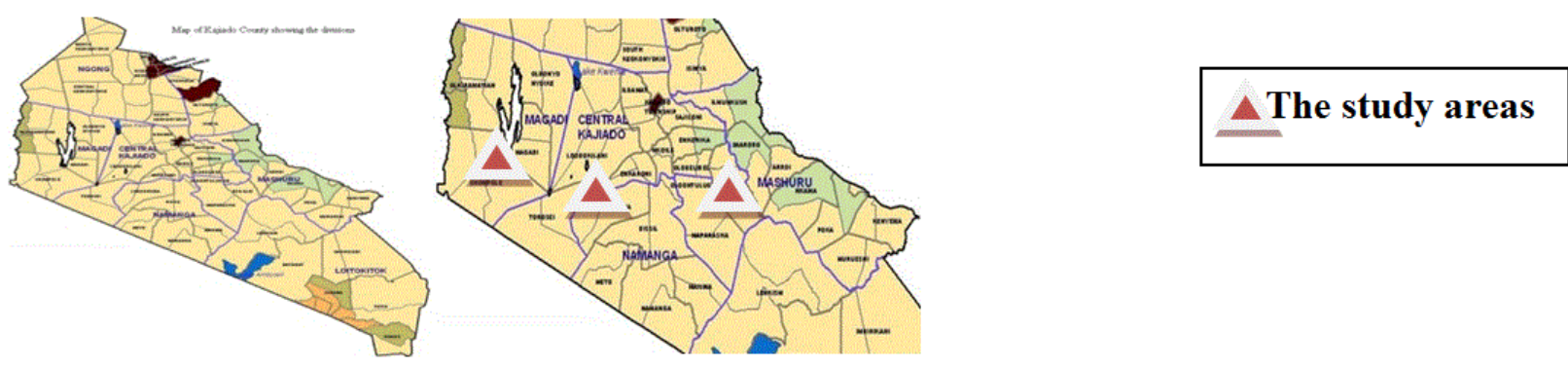

Figure 1: Map of the whole Kajiado County and the study sites.

\section{Diagnostic Tests}

\section{Competitive enzyme linked immunosorbent assay (ELISA) for brucellosis}

Competitive ELISA kit (COMELISA, Veterinary Laboratories Agency, UK) was used. The kit is standardized for the diagnosis of brucellosis in ruminants and human. The reagents were prepared and the tests carried out as per the instructions of the manufacture. The optical densities (OD) were measured at $450 \mathrm{~nm}$ in a microplate photometer (Hum reader, model 18500/1, Awareness Technology Germany). Sera and controls were run in duplicates to compare the two OD readings for every sample. Positive results were denoted by lack of colour development. A positive/negative cut-off was calculated according to the manufacturer's recommendations of $60 \%$ of the mean of the optical density (OD) of the four conjugate control wells. Any test sample giving an optical density equal to or below this value was recorded as being positive. Each plate had six wells for positive control and another six wells for the negative control. In this study, cELISA was used as a confirmatory test and therefore any sera testing positive on this test was regarded as positive. The brucellosis prevalence was calculated based on this test using the formula; Prevalence in $\%=$ (Total number positive/ Total number of samples) x 100 .

\section{Test for Q Fever}

\section{Indirect multispecies enzyme linked immunosorbent assay (ELISA)}

ID Screen ${ }^{\circledR}$ Q-fever Indirect Multi-species kit (IDVET innovative diagnostics, Lillidale Diagnostics England) was used. The kit is standardized for the diagnosis of Q-fever in cattle, sheep and goats.

For each sample the sample percentage was calculated as;

$\mathrm{s} / \mathrm{p}=$ ODsample - ODnc / ODpc-ODnc X 100 . The results were interpreted as follows;

\begin{tabular}{|c|c|}
\hline \multicolumn{2}{|l|}{ Serum } \\
\hline Result & Status \\
\hline$S / P \leq 40 \%$ & Negative \\
\hline $40 \%<\mathrm{S} / \mathrm{P}<50 \%$ & Doubtful \\
\hline $50 \%<\mathrm{S} / \mathrm{P} \leq 80 \%$ & Positive \\
\hline$S / P>80 \%$ & Strongly positive \\
\hline
\end{tabular}

Table 1: Test on Leptospirosis in cattle only.

The reagents were prepared and the tests carried out as per the instructions of the manufacture. The optical densities (OD) were measured at $450 \mathrm{~nm}$ in a micro plate photometer (Hum reader, model 18500/1, Awareness Technology Inc. Germany). Sera and controls were run in duplicates to compare the two OD readings for every sample. The resulting coloration depends on the quantity of specific antibodies present in the specimen to be tested. In the presence of antibodies (Positive), a blue solution appears which becomes yellow after addition of stop solution. In the absence of antibodies (Negative), no coloration appears.

Test on Leptospirosis in cattle is shown in (Table 1) only the test was validated if the mean value of the positive control OD (ODpc was greater than 0.350 and the ratio of the mean OD values of the Positive and Negative control (ODpc/ODnc) is greater than 3. PrioCHECK ${ }^{\circ} \mathrm{L}$. hardjo Ab kit (Prionics Lelysted, Netherlands) was used. The kit is standardized for in vitro detection of antibodies directed against Leptospira interrogans serovars hardjo in serum and milk of cattle. The reagents were prepared and the tests carried out as per the instructions of the manufacture. The optical densities (OD) were measured at $450 \mathrm{~nm}$ in a microplate photometer (Hum reader, model 18500/1, Awareness Technology inc..Germany). Sera and controls were run in duplicates to compare the two OD readings for every sample.

The test was read and the results were calculated by measuring the optical density (OD) of the wells at $450 \mathrm{~nm}$ within 15 minutes of 
Citation: Nakeel MJ, Arimi SM, Kitala PK, Nduhiu G, Njenga JM, et al. (2016) A Sero-epidemiological Survey of Brucellosis, Q-Fever and Leptospirosis in Livestock and Humans and Associated Risk Factors in Kajiado County-Kenya. J Trop Dis 4: 215. doi: 10.4172/2329-891X.1000215

Page 4 of 8

stopping the colour development. The mean OD450 value of the blanks was calculated. The corrected OD450 value of all samples was calculated by subtracting the mean OD450 of the blanks. The percentage positivity (PP) of the reference samples 2, 3 and the test samples were calculated as follows; the corrected OD450 of all samples were expressed as percentage positivity (PP) relative to the corrected mean OD450 value of reference serum;

$\mathrm{PP}=[$ corrected OD450 test sample / corrected OD450 Reference serum $1 \times 100$.

The results were validated if the mean OD450 of the blanks was < 0.150 , the corrected OD450 of reference serum 1 was $>1.000$, the mean PP of reference serum 2 was $<20$ and the mean PP of reference serum 3 was between 20 and 60 . The results were interpreted as follows

\section{Data management and analysis}

All the data obtained from the field was recorded in the notebook and later entered into a computer using Microsoft Excel for serum analysed data and SPPS v20 for questionnaire data for ease of handling. The data was analysed and descriptive statistics of continuous variables done. Association between the explanatory (independent) variables and the outcomes or dependent variables (Prevalence of Brucellosis and Q-fever in cattle sheep goats and human and Leptospirosis in cattle) were investigated by logistic regression using SPSSvs 20, IBM. The size and extent of these three zoonotic diseases in the Kajiado ecosystem remains largely unknown. It was therefore necessary to conduct investigations aimed at establishing their status in order to lessen the potential negative impacts they exert in the ecosystem. In this study the estimation of the prevalence of brucellosis and Q-fever and Leptospirosis was made in cattle, sheep, goats and humans in three Sub- Counties that make a transect from east to west of Kajiado County.

The relationship between each explanatory variable and the outcome variable was investigated and any variable that was significantly associated at the $\mathrm{p}<0.05$ level was included in the multivariate models through forward and backward elimination, the most parsimonious models in which all explanatory variables remained significant at the $\mathrm{p}<0.05$ level was generated. The resulting coloration depends on the quantity of specific antibodies present in the specimen to be tested. In the presence of antibodies (Positive), a blue solution appears which becomes yellow after addition of stop solution. In the absence of antibodies (Negative), no coloration appears.

The Z-test for independent samples was used to determine whether the proportions of animals positive for Brucella, C.burnetii, Leptospira and antibodies their or a combination of three or all the organisms differed significantly between Mashuru, Central and Magadi areas for livestock, and Sultan- Hamud, Kajiado, and Magadi hospitals for humans [15]. The null hypotheses were; Brucellosis, Q-fever and Leptospirosis do not occur in livestock and humans in Kajiado County and there are no risk factors to livestock and humans contracting the three zoonosis in Kajiado County.

\section{Results}

A total of 20 farms and watering points (from Mashuru Kajiado central and Magadi areas) comprising of 248 cattle 305 sheep and 316 goats were investigated for brucellosis and Q-Fever during the study period between July and September 2012. The Result estimated is shown in (Table 2).

\begin{tabular}{|c|c|c|c|c|c|c|c|}
\hline Risk factor & Group & Number & Prevalence & Rate $\%$ & $95 \% \mathrm{Cl}$ & p-value & OR \\
\hline \multicolumn{8}{|l|}{ Human brucellosis and Q-Fever } \\
\hline \multirow{2}{*}{ Age } & $5-14$ years & 14 & 2 & -14.3 & \multirow{2}{*}{$0.9-1.0$} & \multirow{2}{*}{0.06} & \multirow{2}{*}{1.02} \\
\hline & Above 15 years & 223 & 82 & -36.8 & & & \\
\hline \multirow{7}{*}{ Occupation } & Housewife & 120 & 50 & -41.7 & \multirow{7}{*}{$0.6-0.1$} & \multirow{7}{*}{0.05} & \multirow{7}{*}{0.82} \\
\hline & Business lady & 14 & 4 & -28.6 & & & \\
\hline & Teacher & 7 & 2 & -28.6 & & & \\
\hline & Student & 32 & 11 & -34.4 & & & \\
\hline & Pastoralist & 50 & 12 & -24 & & & \\
\hline & Hotelier & 5 & 1 & -20 & & & \\
\hline & Farmer & 9 & 4 & -44 & & & \\
\hline \multirow{2}{*}{ Method of processing meat } & Cooked & 194 & 71 & -36.6 & \multirow{2}{*}{$0.07-0.71$} & \multirow{2}{*}{0.011} & \multirow{2}{*}{0.22} \\
\hline & Roasted & 236 & 13 & -30.3 & & & \\
\hline \multirow{3}{*}{ Do they process blood } & Yes & 5 & 1 & -20 & \multirow{3}{*}{$2.52-32.0$} & \multirow{3}{*}{0.001} & \multirow{3}{*}{8.99} \\
\hline & No & 204 & 66 & -32.4 & & & \\
\hline & Sometimes & 8 & 17 & -60.7 & & & \\
\hline Contact with aborted foetuses & Yes & 8 & 6 & -75 & $0.008-0.77$ & 0.029 & 0.08 \\
\hline
\end{tabular}


Citation: Nakeel MJ, Arimi SM, Kitala PK, Nduhiu G, Njenga JM, et al. (2016) A Sero-epidemiological Survey of Brucellosis, Q-Fever and Leptospirosis in Livestock and Humans and Associated Risk Factors in Kajiado County-Kenya. J Trop Dis 4: 215. doi: 10.4172/2329-891X.1000215

Page 5 of 8

\begin{tabular}{|c|c|c|c|c|c|c|c|}
\hline & No & 229 & 78 & -34.1 & & & \\
\hline \multirow{2}{*}{ Contact with dust } & Yes & 123 & 31 & -25.2 & \multirow{2}{*}{$1.39-6.47$} & \multirow{2}{*}{0.005} & \multirow{2}{*}{3} \\
\hline & No & 114 & 53 & -46.5 & & & \\
\hline \multirow{4}{*}{$\begin{array}{l}\text { Method of disposal of aborted } \\
\text { foetuses }\end{array}$} & Throw into bush & 7 & 3 & -5.4 & \multirow{4}{*}{$0.9-3.8$} & \multirow{4}{*}{0.028} & \multirow{4}{*}{0.11} \\
\hline & Bury/burn & 4 & 0 & 0 & & & \\
\hline & Give to dogs & 37 & 2 & -5.4 & & & \\
\hline & others & 154 & 6 & -3.9 & & & \\
\hline \multirow{2}{*}{ Consume milk } & Yes & 233 & 81 & -34.7 & \multirow{2}{*}{$0.82-162.5$} & \multirow{2}{*}{0.07} & \multirow{2}{*}{11.6} \\
\hline & No & 4 & 3 & -75 & & & \\
\hline \multirow{2}{*}{ Contact with contaminated water } & Yes & 128 & 33 & -25.8 & \multirow{2}{*}{$0.9-3.8$} & \multirow{2}{*}{0.09} & \multirow{2}{*}{1.86} \\
\hline & No & 109 & 51 & -46.8 & & & \\
\hline \multicolumn{8}{|l|}{$\begin{array}{l}\text { Livestock brucellosis and Q-fever } \\
\text { and Leptospirosis }\end{array}$} \\
\hline \multirow{5}{*}{ Water source } & Water pan & 12 & 2 & -16.7 & \multirow{5}{*}{$.052-1.1$} & \multirow{5}{*}{0.07} & \\
\hline & Borehole & 47 & 5 & -10.6 & & & \\
\hline & River & 7 & 0 & & & & \\
\hline & Dam & 14 & 0 & & & & \\
\hline & Borehole and water pan & 8 & 0 & & & & \\
\hline \multirow{2}{*}{ Introduction of new animals } & Yes & 37 & 18 & -48.6 & \multirow{2}{*}{$.87-6.4$} & \multirow{2}{*}{0.09} & \\
\hline & no & 51 & 32 & -62.7 & & & \\
\hline \multirow{2}{*}{ Grazing system } & Individual grazing & 52 & 33 & -63.5 & \multirow{2}{*}{$.01-1.04$} & \multirow{2}{*}{0.005} & \\
\hline & Communal grazing & 36 & 17 & -47.2 & & & \\
\hline
\end{tabular}

Table 2: The results of logistic regression in the study area are presented which showed that among the potential risk factors considered in human sero-prevalence in the study hospitals, occupation $(\mathrm{p}<0.05)$, method of processing meat $(\mathrm{p}<0.01)$, whether they process blood before taking $(\mathrm{p}<0.001)$, contact with aborted foetuses $(\mathrm{p}<0.029)$, contact with dust $(\mathrm{p}<0.005)$ and method of disposal of aborted foetuses and placentas $(\mathrm{p}<0.028)$. The age of the patients was slightly significant $(\mathrm{p}<0.06)$ while the contact with contaminated water and whether they consume milk or not were not significant.

At the same time 250 patients were investigated from three health facilities (Sultan-Hamud, Kajiado referral and Magadi hospitals).

Details of number of both livestock and human samples and Questionnaires collected are shown in (Table 3).

\begin{tabular}{|l|l|l|l|l|l|}
\hline \multirow{2}{*}{ Species } & \multicolumn{5}{|l|}{ Leptospirosis } \\
\cline { 2 - 7 } & Questionnaire data & \multicolumn{5}{l|}{} \\
\hline Cattle & Serum samples & Indirect ProCHECK Lhardjo Ab ELISA & Positive samples\% \\
\hline Mashuru & 39 & 81 & 17 & -6.9 & -8.5 \\
\hline Kajiado & 29 & 94 & 21 & -5.6 & -21.8 \\
\hline Magadi & 20 & 73 & 14 & 54 & \\
\hline Total & 88 & 248 & 54 & & \\
\hline
\end{tabular}

Table 3: Livestock: Management, Introduction of new stock, level of awareness on zoonosis, Frequency of contact with veterinary staff. 
Citation: Nakeel MJ, Arimi SM, Kitala PK, Nduhiu G, Njenga JM, et al. (2016) A Sero-epidemiological Survey of Brucellosis, Q-Fever and Leptospirosis in Livestock and Humans and Associated Risk Factors in Kajiado County-Kenya. J Trop Dis 4: 215. doi: 10.4172/2329-891X.1000215

Page 6 of 8

\section{Serological Results}

Blood samples were obtained from 28/236 (12\%) of persons eighteen years and below ( $\leq 18$ years), 149/236 (63\%) of humans aged $19-45$ years, and 59/236 (25\%) of those above 45 but less than 60 years. The sex of these respondents were female $163 / 236$ (69\%) and 73/236 (31\%) were male. Brucellosis serology results were available for 150 humans and 73 cattle, 69 sheep and 67 goats. A total of 90 human and 156 cattle, 80 sheep and 83 goat sera were tested for Q-fever. Complete questionnaire data was available for 236 humans and 88 livestock.

Results indicated a low overall prevalence of brucellosis in humans $1.3 \%(2 / 150)$ and a moderate overall prevalence in livestock of $12.91 \%$ (27/209). The prevalence estimates in cattle, sheep and goats were $21.92 \%(16 / 73), 8.6 \%(6 / 69)$ and $7.3 \%$ (5/67) for brucellosis .In humans the overall prevalence was found to be $1.33 \%$ with $1.75 \%$ in Kajiado district hospital, $1.47 \%$ in Sultan Hamud hospital and $0 \%$ in Magadi hospital. In Bovine, the highest prevalence was Mashuru area with $40 \%$, followed by Magadi area with $19.04 \%$ but $0 \%$ in Kajiado area. The overall prevalence in the study area was found to be $21.92 \%$. In Ovine, the overall prevalence in the study area was $8.69 \%$, with $14.8 \%$ in Mashuru, $5 \%$ in Kajiado and $4.5 \%$ in Magadi area. In Caprine, the overall prevalence was found to be $7.25 \%$, with $3.30 \%$ in Mashuru, $10 \%$ in Kajiado and $11.8 \%$ in Magadi areas respectively.

The highest prevalence of Q-fever in humans was found to be $32 \%$ in Sultan Hamud hospital in Mashuru district, with 25\% in Kajiado District Hospital in Kajiado district and 22\% in Magadi Hospital serving residents in Magadi division. The overall prevalence of Q-fever in humans was found to be $26.7 \%$.In Bovine the overall prevalence was found to be $89.7 \%$ with $88.5 \%$ in Mashuru, $85.2 \%$ in Kajiado and $92 \%$ in Magadi. In Ovine the overall prevalence was found to be $57.5 \%$ with $58.8 \%$ in both Mashuru and Kajiado and 55.2\% in Magadi. The overall prevalence of Q-fever in Caprine was found to be $83.1 \%$, with $79.4 \%$ in Mashuru, $86.2 \%$ in Kajiado and $85 \%$ in Magadi. The prevalence of Leptospirosis based on Indirect ELISA was found to be $21.8 \%(54 / 248)$ in cattle only. The prevalence was highest in cattle found in central sub county at $8.5 \%, 6.9 \%$ in Mashuru Sub County and 5.6\% in Magadi Sub County.

\section{Questionnaire surveys}

As presented in table 5 the prevalence of the two diseases was higher in persons 15 years and above 82/223 (36.8\%) than persons 5-14 years old and occupation of being a housewife had a high prevalence of $50 / 120(41.6 \%)$ than all the other occupations. Cooking meat had $71 / 194$ (36.6\%) being sero positive than roasting meat. A high number of respondents that used blood without processing 66/120 (32.4\%) showed that this factor was significant in testing positive. Pastoralists that threw away foetal discharges and aborted foetuses in the bush were many at $3 / 7(5.4 \%)$ as those giving it to the dogs $2 / 37(5.4 \%)$.The data on livestock collected indicated that the majority of the respondents watered their livestock using borehole water (47) in dams (14) and water pans (12). Even though the pastoralists that practised communal grazing $17(47.22 \%$ were slightly less than those that practiced individual grazing $33(63.46 \%)$ those in individual grazing showed a higher prevalence.

\section{Discussions}

It is estimated that around $61 \%$ of the known 1415 human pathogens are zoonotic [16]. The concept of 'one medicine' which is defined as the science of all human and animal health diseases has been around for several decades but its uptake is still lacking in many developing countries where it could have most impact. Understanding the epidemiology of diseases such as brucellosis, and Q-fever are important veterinary issues relating to production losses and abortions. However, the zoonotic nature of these diseases means that it is also important for the medical profession to understand the extent and prevalence of these diseases in the livestock reservoir. The two diseases produce very variable non-specific symptoms in people and are generally believed to be hugely under reported largely due to confusion with malaria in developing countries where $50-80 \%$ of malaria cases may suffer fevers resulting from other causes [17].

\section{Brucellosis and Q-fever in Human}

In this study, the overall sero-prevalence of brucellosis in humans was low at $1.3 \%(2 / 150)$ in the sampled patients in the three hospitals of Sultan Hamud, Kajiado referral and Magadi. Kajiado hospital showed a prevalence of $1.75 \%$ (1/57), $1.47 \%(1 / 68)$ in Sultan Hamud and $0 \%(0 / 25)$ in Magadi. The prevalence differed from other findings in the neighbouring counties of Narok $12 \%$ Turkana County $17 \%$ [18]. The low prevalence in this study site is not consistent with other findings which show that the disease is more prevalent among nomadic pastoralists as Kajiado County is still being classified as nomadic pastoral area [19]. The questionnaire data showed that despite the people being thought to be more exposed because of keeping large herds of livestock, consuming animal products, using animals by products like skins and manure and living in close association the prevalence of brucellosis was found to be low and not high as expected This is despite the high prevalence recorded of cases of diagnosed brucellosis on RBPT in Kajiado of 609 from year 2007- 2012 [17].

The prevalence of Q-fever on the other hand was found to be high at an overall of 24/90 (26.7\%) despite the complete lack of awareness in the study area comparable to $30.9 \%$ prevalence on achieved patients in western Kenya [9]. No hospital ever made an effort to suspect Q-fever despite the common symptoms it shows with other diseases like Malaria, Typhoid and brucellosis. The highest prevalence was recorded in Sultan Hamud hospital of (32\%) followed by Kajiado at (25\%) and lowest at Magadi hospital (22\%). In addition to sero-prevalence, this study was also carried out to determine the risk factors associated with disease occurrence in humans in this transect of the county. There was a high level of awareness among the respondents on brucellosis with $61 \%(\mathrm{n}=142$ on questionnaire data) naming the disease as "ugonjwa wa Maziwa" transmitted though consumption of raw milk, even though they were aware that it is also associated with meat, while those that were not aware were half at $33 \%(n=81)$ with only $6 \%(n=13)$ being partially aware. There was totally no awareness of Q-fever among veterinary staff, health staff and the respondents probably because they have not heard of the disease $[20,21]$. The pastoralists do not relate brucellosis to abortion, retained placenta, assisting animals during parturition, handling of aborted foetuses and throwing of foetal membranes in the bush or giving to dogs. This makes them handle abortion materials and placenta without any protection or hygienic consideration including hand washing which is not routinely practised due to shortage of water.

Among the respondents to both diseases in humans $82 \%(n=195)$ had very close contact with animals, sharing compounds, watering points and premises with neonates posing a high risk to infection and showed a prevalence of $33.7 \%$ and $30.2 \%$ of brucellosis and Q-fever respectively. On occupation $53 \%(n=120)$ of the patients interviewed were housewives in pastoral homes that showed a prevalence of $41.6 \%$ 
(50/120) while $20 \%(n=45)$ were pastoralist men that had a prevalence of $24 \%(12 / 50)$. Students of ages $5-18$ years had a high prevalence of brucellosis of $34.4 \%(11 / 32)$ and $33.3 \%(12 / 36)$ prevalence of Q-fever [22].

Laboratory tests are important in proper diagnosis for both diseases but more specifically for brucellosis in both humans and animals. Studies have highlighted challenges encountered in remote areas in carrying out laboratory diagnosis [5]. In humans the two diseases present signs and symptoms similar to other flu-like conditions such as malaria, typhoid, streptococcal infections and rheumatic fever [17].

There was no significant difference in the sero-prevalence in the three hospitals among the patients sampled. The prevalence of the two diseases in humans could be much higher than was portrayed by the hospital record. This is because testing is not carried out consistently in the hospitals and most patients are tested for malaria and typhoid only and the other diseases are only when the patient does not respond to treatment against malaria or typhoid. Lack of reagents was also witnessed in the hospitals coupled with inadequately trained staff to carry out quick testing to take care of the high number of patients turning up especially during market days. Health facilities are located away from the people with the nearest facilities being $80 \mathrm{kms}$ away for most patients [6]. The tests are also carried out at a cost making it unaffordable for many patients.

\section{Brucellosis and Q-fever and Leptospirosis in livestock}

The prevalence of brucellosis in cattle in the three districts was highest in Kajiado East (Mashuru) at 40\% (12/30) followed by Kajiado West (Magadi) at $19.04 \%$. Kajiado Central Sub County (Central) had no positive tests on cattle making the overall prevalence to stand at $21.9 \%$ across transect of the county. The overall prevalence in ovines was found to be higher in Mashuru too at $14.8 \%$ (4/27), 5\% (1/20) and $4.5 \%(1 / 22)$ in Magadi district. Magadi on the other hand had the highest prevalence of brucellosis in goats at $11.8 \%(2 / 17)$, then Kajiado at $10 \%(2 / 20)$ and lowest in Mashuru at 3.3\% (1/30). The prevalence of brucellosis was highest in cattle (21.9\%) and lowest in goats at (7.25\%). This could be attributed to the fact that goats are browsers and therefore feed on less contaminated pasture than cattle and sheep.

Q-fever prevalence in livestock was high in all the three species across all the three districts much higher than found in recent studies done in 2007-2008 in western Kenya. The overall prevalence in cattle was found to be $89.7 \%(140 / 156)$ higher than $7-57 \%$ as reported by [10]. The overall prevalence in sheep in this study was $57.5 \%(46 / 80)$ compared to $18.2 \%(n=159)$ in the same survey and $83.1 \%(69 / 83)$ compared to $32 \%(n=378)$ in goats. The prevalence of Leptospirosis based on Indirect ELISA was found to be $21.8 \%(54 / 248)$ in cattle only. The prevalence was highest in cattle found in central sub county at 8.5 \%, 6.9\% in Mashuru Sub County and 5.6\% in Magadi Sub County.

The likely explanation for this rather high prevalence could lie in the lack of awareness on zoonotic diseases, large numbers of animals kept, close contact of animals being herded and housed together and the sharing of watering places as opposed to better awareness, low number of animals, keeping on type of animal and individual grazing and watering of animals in western Kenya. The sharing of enclosures and grazing areas between calving animals and the others could be causing a build-up of the Coxiella in Kajiado livestock. Majority of the livestock owners interviewed said they had encountered cases of abortion and retained placenta within in livestock where the aborted foetuses are thrown to the bush or given to dogs spreading the organisms further.
The low brucellosis sero-prevalence was unexpected based on previous studies from the literature. The high sero-prevalence of exposure to Coxiella burnetti represent a major challenge both from a veterinary and a public health view point. It is likely that there is a high incidence of abortion/reproductive failure in affected herds leading to potentially high levels of exposure of livestock owners and their families which is then not being correctly diagnosed. Further studies are clearly needed to study these important zoonoses and to be able to understand the human and animal interactions and the clinical significance of these sero-prevalence in both the animal and for the human populations. Domestic ruminants are sources of human infection, through direct contact or contamination of the environment during parturition or abortion. Although not assessed in this study, consumption of dairy products from infected ruminants is also likely to pose a risk. Although risk factor studies would be helpful to identify the principal modes of transmission to humans in this setting, our results support the likely efficacy of recognized public health and hygiene measures for mitigating the risk of transmission from domestic ruminants, such as confinement of domestic animals during parturition, avoidance of contaminated pastures and contact with placental material, and boiling or pasteurization of milk before consumption. This study also suggests that investigation of the role of domestic dogs and tick-borne transmission is warranted to identify potential sources of infection and risk factors that are currently not well recognized [3].

\section{Conclusions}

There is a high likelihood that brucellosis and Q-fever are enzootic in the study area and present a serious public health problem among the inhabitants of the county.

It should be noted that while abortions have been associated with better known brucellosis but Q-fever could be contributing significantly to these causing abortions.

Both zoonoses appear to be of major public health importance in humans and animals which calls for concerted efforts in combating them in the spirit of one health approach between the veterinary and health personnel.

Investigations on brucellosis and Q-fever in livestock have virtually not been done and therefore no control measures have been put in place.

Some health centres carry out limited tests for brucellosis but face shortages of test reagents and records are inconsistent and unreliable.

A substantial number of people lack awareness on the risk factors of the two diseases and the methods of prevention and control.

\section{Recommendations}

The veterinary personnel in Kajiado County should make an effort to investigate all cases of abortions and retained placentas that are included in their disease surveillance reports. This calls for strengthening laboratory diagnostic capacity in the county by training more veterinary and health staff and providing diagnostic equipment and reagents.

Creating awareness among the people on the seriousness of the causes, modes of transmission, risk factors and methods of prevention of the two diseases should be undertaken as soon as possible.

Slaughter house workers and other veterinary personnel should wear protective gear when in contact with fresh animal tissues. 
Citation: Nakeel MJ, Arimi SM, Kitala PK, Nduhiu G, Njenga JM, et al. (2016) A Sero-epidemiological Survey of Brucellosis, Q-Fever and Leptospirosis in Livestock and Humans and Associated Risk Factors in Kajiado County-Kenya. J Trop Dis 4: 215. doi: 10.4172/2329-891X.1000215

Page 8 of 8

Effort should be made by health personnel to do a confirmatory diagnosis of all diseases presenting flu-like symptoms before treatment to avoid misdiagnosis, drug misuse and long suffering of patients.

\section{References}

1. Cleaveland S, Laurenson MK, Taylor LH (2001) Diseases of humans and their domestic mammals: pathogen characteristics, host range and the risk of emergence. Philos Trans R Soc Lond B Biol Sci 356: 991-999.

2. Taylor LH, Latham SM, Woolhouse ME (2001) Risk factors for human disease emergence. Philos Trans R Soc Lond B Biol Sci 356: 983-989.

3. Haydon DT, Cleaveland S, Taylor LH, Laurenson MK (2002) Identifying reservoirs of infection: a conceptual and practical challenge. Emerg Infect Dis 8: $1468-1473$.

4. Scolamacchia F, Handel IG, Fèvre EM, Morgan KL, Tanya VN, et al. (2010) Serological patterns of brucellosis, leptospirosis and Q fever in Bos indicus cattle in Cameroon. PLoS One 5: e8623.

5. McDermott JJ, Arimi SM (2002) Brucellosis in sub-Saharan Africa: epidemiology, control and impact. Vet Microbiol 90: 111-134.

6. Ministry of Health Annual Reports Kajiado District Hospital, 2007- 2012.

7. Kangethe EK, Arimi SM, Omore AO, Mcdermott JJ, Nduhiu JG, et al. (2000) The prevalence of antibodies to Brucella abortus in marketed Milk in Kenya and its Public Health Implications.

8. Rodolakis A, Berri M, Héchard C, Caudron C, Souriau A, et al. (2007) Comparison of Coxiella burnetii shedding in milk of dairy bovine, caprine, and ovine herds. J Dairy Sci 90: 5352-5360.

9. Knobel DL, Maina AN, Cutler SJ, Ogola E, Feikin DR, et al. (2013) Coxiella burnetii in humans, domestic ruminants, and ticks in rural western Kenya. Am J Trop Med Hyg 88: 513-518.

10. Madariaga MG, Rezai K, Trenholme GM, Weinstein RA (2003) Q fever: a biological weapon in your backyard. Lancet Infect Dis 3: 709-721.
11. Mediannikov O, Fenollar F, Socolovschi C, Diatta G, Bassene H, et al. (2010) Coxiella burnetii in humans and ticks in rural Senegal. PLoS Negl Trop Dis 4: 654.

12. Malavika P, Nicholson WL, Aubree JR, Gilbert J, Itzpatrick A, et al. (2011) Q-fever, spotted fever group and Typhus group rickettsioses among hospitalized febrile patients in Northern Tanzania. Clin Infect Dis 53: 8-15.

13. Levett PN (2001) Leptospirosis. Clin Microbiol Rev 14: 296-326.

14. Hunter P, Herr S (2004) Infectious Diseases of Livestock. (2ndedtn) Oxford University Press, volume 3, chapter 136.

15. Dohoo I., Wayne M, Styhn H (2010) Veterinary Epidemiologic Research. Veterinary Epidemiology Research Textbooks.

16. Cloeckaert A, Verger JM, Grayon M, Paquet JY, Garin-Bastuji B, et al. (2001) Classification of Brucella spp. isolated from marine mammals by DNA polymorphism at the omp2 locus. Microbes Infect 3: 729-738.

17. Maichomo MW, McDermott JJ, Arimi SM, Gathura PB, Mugambi TJ, et al. (2000) Study of brucellosis in a pastoral community and evaluation of the usefulness of clinical signs and symptoms in differentiating it from other flu-like diseases. Afr J Health Sci 7: 114-119..

18. Nanyende DW (2009) Prevalence of Brucellosis in Northern Turkana. Thesis Document.

19. Schelling E, Diguimbaye C, Daoud S, Nicolet J, Boerlin P, et al. (2002) Brucellosis and Q-fever sero-prevalence of nomadic pastoralists and their livestock in Chad Preventive Veterinary Medicine 4: 279-293.

20. Kajiado County (2007-2012) MOH annual reports, Kajiado District Hospital.

21. Director of Veterinary Sevices, Kenya Annual Reports (2003- 2011).

22. Kajiado District Strategic Plan (2005-2010) for Implementation of the National PopulationPolicy for Sustainable Development. Prevalence of Brucellosis in Northern Turkana. Thesis Document. 